\title{
PCLO rs2522833 impacts HPA system activity in healthy young adults
}

\author{
C Kuehner ${ }^{1}$, S Huffziger ${ }^{1}$, SH Witt ${ }^{2}$ and M Rietschel ${ }^{2}$
}

Recent genetic studies showed evidence for a role of the single-nucleotide polymorphism rs2522833 within the PCLO gene in the etiology of major depression, and rs2522833 has been shown to modulate hypothalamic pituitary adrenal (HPA) axis activity during antidepressant treatment. Monoaminergic modulation of the HPA system may be one possible pathomechanism by which PCLO exerts its effect on depression. In the present study, we investigated the effect of rs2522833 on the cortisol awakening response (CAR) in healthy young adults. A total of 66 healthy volunteers from the community ( 36 men and 30 women) aged 18-25 years without individual or family history of affective disorders and schizophrenia collected saliva cortisol samples at $0,30,45$ and $60 \mathrm{~min}$ after awakening on two consecutive working days. We identified a blunted CAR (AUCinc) in rs2522833 risk-allele (C) carriers, possibly indicating exhausted regulatory mechanisms underlying the HPA system. We also identified higher neuroticism scores in rs2522833 risk-allele carriers but no phenotypic correlation between the CAR (AUCinc) and neuroticism. These findings suggest that the rs2522833 risk variant might increase vulnerability to depression both by physiological and behavioral pathways, which appear, however, not to be substantially overlapped. Replication with larger samples is warranted. Translational Psychiatry (2011) 1, e10; doi:10.1038/tp.2011.11; published online 31 May 2011

\section{Introduction}

A recent genome-wide association study ${ }^{1}$ showed evidence for a role of the single-nucleotide polymorphism rs2522833 within the piccolo (PCLO) gene in the etiology of major depression, which was supported by a subsequent meta-analysis ${ }^{2}$ and joint reanalysis of correlated multiple single-nucleotide polymorphisms. ${ }^{3}$ Another region in PCLO has been associated with bipolar disorder. ${ }^{4}$ Furthermore, rs2522833 modulated hypothalamic pituitary adrenal (HPA) axis activity during antidepressant treatment. ${ }^{5}$ In this report, we show that rs2522833 impacts HPA axis regulation in healthy young adults.

The protein product of the PCLO gene is relevant for monoaminergic neurotransmission in the brain, making it a plausible candidate gene for affective disorders. Although the original genome-wide association study ${ }^{1}$ and the subsequent meta-analysis ${ }^{2}$ replicated positive findings only in populationbased samples, rs2522833 influence on HPA system response to antidepressants has been observed in depressed patients, referred as inpatients. ${ }^{5}$ Here, C-allele carriers showed larger HPA dysregulation than AA-carriers. This study suggests monoaminergic modulation of the HPA system as one possible pathomechanism by which PCLO exerts its effect on depression.

In the present study, we sought to investigate the effect of rs2522833 on HPA system activity in healthy young adults. Investigating healthy samples allows to measure effects of common gene variants on physiological and behavioral phenotypes, which are not yet confounded with but possibly increase vulnerability to depression. As a physiological marker, we investigated the cortisol awakening response (CAR), denoting the rise in cortisol levels during the first hour after awakening. The CAR reflects the sensitivity of the HPA axis in response to a naturally occurring stressor (awakening) and has gained growing attention because of its distinct association with stress and psychopathology, ${ }^{6}$ and its higher genetic liability than daytime cortisol levels. ${ }^{7}$ In depression, both increased and blunted CARs have been identified, ${ }^{7}$ possibly because of different subgroups and/or severity of illness. A recent meta-analysis that also included community studies concluded that the CAR is negatively correlated with depression, especially its dynamic component. ${ }^{7}$ A blunted morning rise in cortisol levels has also been identified in healthy subjects with cognitive vulnerability to depression. ${ }^{8}$ Therefore, we expected particularly the dynamic aspect of the CAR to be lower in C-allele carriers.

In parallel to physiological phenotypes, personality traits may underlie the association between rs2522833 and vulnerability to depression. Particularly neuroticism has been shown to have a clear genetic overlap with major depression and to predict the subsequent onset of the disorder. ${ }^{9}$ Furthermore, as neuroticism reflects a tendency to cope poorly with stress, ${ }^{9}$ HPA axis (dys)function may be involved in this association. Therefore, we also aimed to investigate the association

\footnotetext{
${ }^{1}$ Department of Psychiatry and Psychotherapy, Research Group Longitudinal and Intervention Research, Central Institute of Mental Health, University of Heidelberg, Mannheim, Germany and ${ }^{2}$ Department of Genetic Epidemiology in Psychiatry, Central Institute of Mental Health, Mannheim, University of Heidelberg, Mannheim, Germany

Correspondence: Professor C Kuehner, Department of Psychiatry and Psychotherapy, Research Group Longitudinal and Intervention Research, Central Institute of Mental Health, University of Heidelberg, J 5, Mannheim D-68159, Germany.
}

E-mail: Christine.Kuehner@zi-mannheim.de

Keywords: PCLO gene; HPA axis; cortisol awakening response; neuroticism

Received 23 March 2011; accepted 15 April 2011 
between rs2522833 and neuroticism, as well as a possible phenotypic relationship between the CAR and neuroticism.

\section{Subjects and methods}

We recruited 66 healthy volunteers aged 18-25 years (36 men and 30 women) from the community without individual or family history of affective disorders and schizophrenia. On two consecutive working days, participants collected four saliva cortisol samples per day during the first hour after awakening (at awakening, $+30 \mathrm{~min},+45 \mathrm{~min}$ and +60 min). Participants were instructed not to brush their teeth or eat, drink and smoke during sampling periods, and to record exact sampling times and adherence to the protocol.

Saliva samples were analyzed using a time-resolved fluorescence immunoassay. Intra- and interassay variability were $<10 \%$. Cortisol samples for each time point were averaged across days. If subjects did not adhere to the protocol for a probe, these values were excluded from analyses. For the CAR, we calculated both the area under the curve with respect to increase $\left(A \cup C_{i n c}\right)$ and with respect to the ground $\left(A \cup C_{g}\right)$. The $A \cup C_{i n c}$ denotes the dynamic fluctuation of the system from the awakening baseline, whereas $\mathrm{AUC}_{\mathrm{g}}$ is an estimate of total cortisol secretion during the first hour. ${ }^{10}$ Analyses of covariance involving genotype (AA- versus $\mathrm{C}$-allele carriers) as independent factor and CAR components as dependent variables were controlled for depressive symptom levels, sex, age and habitual smoking.

Depressive symptoms were measured with the Beck Depression Inventory, second revision, ${ }^{11}$ and neuroticism was measured with the Neo-Five-Factor Inventory subscale neuroticism. ${ }^{12}$ The analysis of covariance involving neuroticism as dependent variable was controlled for depressive symptoms.

Rs2522833 was genotyped using a TaqMan $5^{\prime}$ nuclease assay. A duplication of $15 \%$ of the sample revealed reproducibility of $100 \%$. Allele frequencies (AA: $30.3 \%, A C$ : $56.1 \%$ and CC: $13.6 \%$ ) were in Hardy-Weinberg equilibrium $(P>0.05)$. Owing to small numbers of CC homozygotes, we pooled subjects with $\mathrm{AC}$ and $\mathrm{CC}$ genotypes (risk-allele carriers, $n=46$ ).

\section{Results and discussion}

Compared with rs2522833 AA-carriers, C-allele carriers showed significantly lower CAR_AUC ${ }_{\text {inc }}$ than AA-carriers $(282.0 \pm 319.1 \quad$ versus $467.0 \pm 269.1, \quad F(1,61)=4.35$, $P=0.042$, see Figure 1$)$, but similar CAR_AUC $(1074.3 \pm 305.2$ versus $1115.7 \pm 368.2, F(1,61)=0.07$, not significant). Furthermore, C-carriers scored higher on neuroticism than $A A$-carriers $(18.6 \pm 6.7$ versus $15.5 \pm 6.7$, $\mathrm{F}(1,63)=4.17, P=0.045)$. However, by adjusting for age, sex and habitual smoking, neuroticism was not correlated with CAR_AUC ${ }_{\text {inc }}\left(r_{\text {partial }}=-0.116\right.$, not significant $)$, and its inclusion as a further covariate in a respective analysis of covariance model did not affect the significant association between rs2522833 genotype and CAR_AUC inc $_{\text {in }}$ (F $(1,60)=4.40, P=0.041)$.

Although these results clearly need replication in larger samples, they point to a possible influence of rs2522833 on
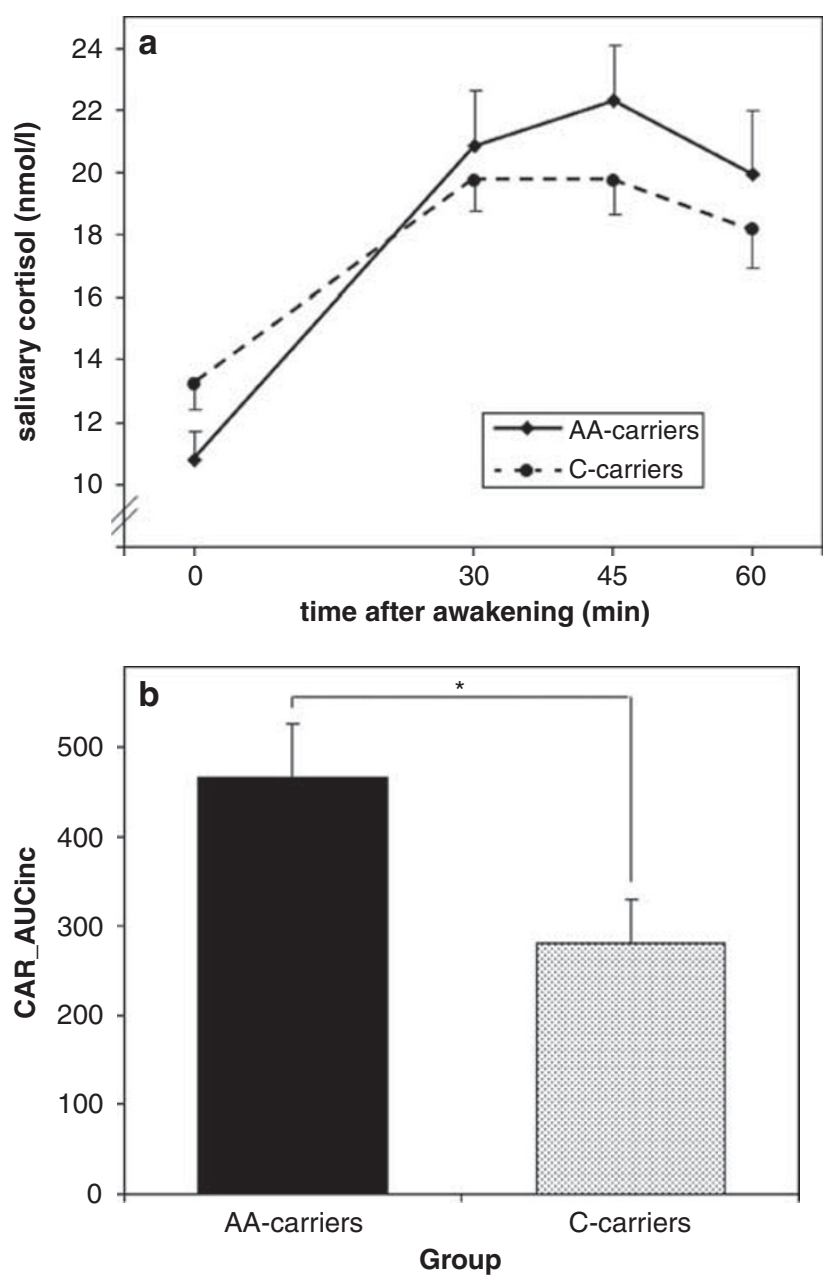

Figure 1 The cortisol awakening response (CAR) for rs2522833 AA- and C-allele carriers. (a) Course of cortisol during the first hour after awakening. (b) The $C A R$ area under the curve with respect to increase $\left(A \cup C_{\text {inc }}\right)$. In both graphs, error bars represent standard errors of the mean. ${ }^{*} P<0.05$.

both HPA activation and neuroticism. These associations were not due to genotype group differences in subclinical symptoms, which were controlled in the respective models. Both dysregulation of the CAR and neuroticism have been identified as vulnerability factors for depression. ${ }^{6-10}$ As hypothesized, rs2522833 was associated particularly with the dynamic component of the $\mathrm{CAR}^{7}$ and the blunted CAR increase in risk-allele carriers might indicate exhausted regulatory mechanisms underlying the HPA system. Furthermore, we identified an association between rs2522833 genotype and neuroticism, but no phenotypic correlation between CAR_AUC ${ }_{\text {inc }}$ and neuroticism. Therefore, our results suggest that the rs2522833 risk variant might increase vulnerability to depression both by physiological and by behavioral pathways, which appear, however, not to be substantially overlapped. Alternatively, unknown factors may have masked the relationship between CAR_AUC ${ }_{\text {inc }}$ and neuroticism. Finally, it is not yet known whether rs2522833 itself or a variant in disequilibrium with it is causally linked to physiological and behavioral phenotypes. 


\section{Conflict of interest}

The authors declare no conflict of interest.

Acknowledgements. This project was supported by the German Research Foundation (DFG KU1464/4-1 and SFB636 D4, Z4).

1. Sullivan PF, de Geus EJC, Willemsen G, James MR, Smit JH, Zandbelt T et al. Genome-wide association for major depressive disorder: a possible role for the presynaptic protein piccolo. Mol Psychiatry 2009; 14: 359-375.

2. Hek K, Mulder $\mathrm{CL}$, Luijendijk HJ, van Duijn $\mathrm{CM}$, Hofman A, Uitterlinden AG et al. The PCLO gene and depressive disorders: replication in a population-based study. Hum Mol Genet 2010; 19: 731-734.

3. Bochdanovits Z, Verhage M, Smit AB, de Geus EJ, Posthuma D, Boomsma DI et al. Joint reanalysis of 29 correlated SNPs supports the role of PCLO/Piccolo as a causal risk factor for major depressive disorder. Mol Psychiatry 2009; 14: 650-652.

4. Choi KH, Higgs BW, Wendland JR, Song J, McMahon FJ, Webster MJ. Gene expression and genetic variation data implicate PCLO in bipolar disorder. Biol Psychiatry 2011; 69 353-359.

5. Schuhmacher A, Mössner R, Höfels S, Pfeiffer U, Guttenthaler V, Wagner M et al. PCLO rs2522833 modulates HPA system response to antidepressant treatment in major depressive disorder. Int J Neuropsychopharmacol 2011; 14: 237-245.
6. Dedovic K, Engert V, Duchesne A, Lue SD, Andrews J, Efanov SI et al. Cortisol awakening response and hippocampal volume: vulnerability for major depressive disorder? Biol Psychiatry 2010; 68: 847-853.

7. Chida Y, Steptoe A. Cortisol awakening response and psychosocial factors: a systematic review and meta-analysis. Biol Psychology 2009; 80: 265-278.

8. Kuehner C, Holzhauer S, Huffziger S. Decreased cortisol response to awakening is associated with cognitive vulnerability to depression in a nonclinical sample of young adults. PNEC 2007; 32: 199-209.

9. Klein DN, Kotov R, Bufferd SJ. Personality and Depression: Explanatory Models and Review of the Evidence. Annu Rev Clin Psychol 2011; 7: 269-295.

10. Pruessner JC, Kirschbaum C, Meinlschmid G, Hellhammer DH. Two formulas for computation of the area under the curve represent measures of total hormone concentration versus time-dependent change. PNEC 2003; 29: 563-564.

11. Hautzinger M, Keller F, Kuehner C. BDI-II Beck Depression Inventory II, German version. Pearson Assessment: Frankfurt, Germany, 2006.

12. Costa PT, McCrae RR. Revised NEO Personality Inventory (NEO-PI-R) and NEO FiveFactor Inventory (NEO-FFI) professional manual. Psychological Assessment Resources: Odessa, USA, 1992.

Translational Psychiatry is an open-access journal published by Nature Publishing Group. This work is licensed under the Creative Commons Attribution-NoncommercialNo Derivative Works 3.0 Unported License. To view a copy of this license, visit http://creativecommons.org/licenses/by-nc-nd/3.0/ 